\title{
NON SURGICAL MANAGEMENT OF CUTANEOUS SINUS TRACT OF DENTAL ORIGIN: A REPORT OF THREE CASES
}

Rohit Kansal ${ }^{1}$, Aishvarya Kaushik², Sangeeta Talwar³, Sarika Chaudhary ${ }^{4}$, Ruchika Nawal ${ }^{5}$

\section{HOW TO CITE THIS ARTICLE:}

Rohit Kansal, Aishvarya Kaushik, Sangeeta Talwar, Sarika Chaudhary, Ruchika Nawal. "Non surgical management of cutaneous sinus tract of dental origin: a report of three cases". Journal of Evolution of Medical and Dental Sciences 2013; Vol. 2, Issue 46, November 18; Page: 9042-9047.

ABSTRACT: Chronic inflammation of the dental pulp is one of the reasons for cutaneous sinus tract of odontogenic origin. A cutaneous sinus or extra oral sinus from a lesion of endodontic origin is rare as compared to an intra oral sinus and may occur as result of long standing inflammatory process associated with necrotic pulp. Such patients are usually healthy and are unaware of the underlying asymptomatic dental problem. Common clinical presentation in these patients is a papule or nodule located most commonly in the chin, cheek or in submandibular area and this leads them to seek treatment from a general physician or a dermatologist. They may undergo unnecessary multiple biopsies, multiple surgical interventions, multiple antibiotic regimens, and even be subjected to radiation therapy or electrodessication. However more often than not, recurrence of sinus tract takes place because the primary dental etiology is never taken care of. The present article aims to report three cases of cutaneous sinus tracts in the submental area which were treated conservatively by endodontic treatment of the involved teeth.

INTRODUCTION: Cutaneous odontogenic sinus tracts often pose a diagnostic dilemma and are often misdiagnosed. Patients with cutaneous sinus tracts often undergo dermatological and other surgical interventions before being referred to the dental surgeon. ${ }^{1}$ Therefore all chronic draining sinus tracts of the face and neck signal the need of a thorough dental evaluation.

Most commonly, the etiology of odontogenic sinus tracts involves endodontically involved teeth with pulpal necrosis and periapical infection. ${ }^{2}$ Following egress from the necrotic pulp canal system, micro organisms and their by products present in the periradicular area might perforate the cortical plate with the infection draining onto the mucosal or cutaneous surface following the path of least resistance. 4,5

Chan et al reported an extraoral cutaneous sinus tract caused by vertical root fracture. ${ }^{6}$ Other differential diagnoses include traumatic lesions, fungal and bacterial infection, neoplasm, presence of foreign body, local skin infection, pyogenic granuloma, chronic tuberculosis lesion, osteomyelitis, actinomycosis and gumma of tertiary syphilis. ${ }^{4,6}$

The present article aims to present two such cases of cutaneous sinus tracts in the chin region, managed conservatively by endodontic treatment of the offending teeth.

Case report 01: A healthy 28 years old male presented to our department with a chief complaint of extraoral nodular growth with intermittent pus discharge on his chin for the past one and a half years. He suffered from no other medical condition and was not on any medication and had no allergies. Past dental history revealed that about two years before the patient had undergone a traffic accident and six months later a nodular growth occurred in his chin area. He also stated that when the lesion started to discharge purulent fluid, he visited a physician who prescribed 
antibiotics. However pus recurred each time the therapy was interrupted. On clinical examination, the mandibular anterior teeth had normal appearance concerning integrity of its crowns. However the mandibular right lateral incisor had slight discoloration and responded negatively to pulp sensitivity test. The rest of the mandibular anterior teeth responded within normal limits. Radiographic examination showed periradicular radiolucency associated with apical region of \# 42 . The clinical diagnosis of chronic apical periodontitis with extraoral sinus was made.

Non surgical endodontic therapy of \# 42 was planned. During the first visit access cavity preparation was done under rubber dam(Hygienic, Coltene, Whaledent) isolation without local anaesthesia. Necrotic content of the pulp chamber and root canal was removed. Working length was determined with apex locator and confirmed radiographically. Biomechanical preparation was done with combined rotary nickel titanium protaper universal and hand instrumentation using crown down technique. During preparation root canals were irrigated using $5.25 \%$ sodium hypochlorite. Calcium hydroxide was used as intracanal medicament. Only one week after nonsurgical endodontic treatment drainage from sinus tract was stopped. Three weeks after first visit the root canal were obturated with $\mathrm{AH}$ plus sealer and corresponding gutta percha by cold lateral condensation technique. After one month patient showed healed sinus tract but had a small depressed area over the healed region.

Case report 2: A 19 year old male was referred from an oral and maxillofacial surgery department with a chief complaint of episodic drainage of pus from the skin in the chin region since 4 months. The medical history was noncontributory. Clinical examination showed draining lesion approximately $1.2 \mathrm{~cm}$ in diameter in the submental area. Palpation elicited an exudate discharge from it. Intraorally, no vestibular swelling was present. An intraoral periapical radiograph showed diffuse radiolucency surrounding mandibular central incisors. A diagnosis of suppurative apical periodontitis was made with need of root canal treatment. After root canal preparation was completed, calcium hydroxide was inserted into the canals, and the patient was recalled after a week. Upon recall, the draining lesion had become smaller in diameter. Root canal treatment was completed and final restoration was done with light cured composite. The patient was recalled after 2 months. Clinically the draining lesion had completely healed with scar formation.

CASE 3: A 20 year old female patient reported to our department with the chief complaint of a swelling in the chin area of the face since 6 months. The patient gave history of recurrence and remission of fever for one month, for which she was treated by a general physician. Since there was recurrence of the lesion and fever over and over again, she was referred for dental opinion. On the day of examination, the patient was afebrile and gave negative history of any systemic disorder. Extraoral examination revealed erythematous nodule with continuous drainage in the submental area. The nodule was soft on palpation and it elicited a purulent discharge. Also, the lesion was present for last 6 months, which was alternately appearing and disappearing. Intra oral examination revealed discoloured mandibular left central incisor with radiographic evidence of periapical pathology. Endodontic treatment was initiated and calcium hydroxide was administered as intracanal medicament. The lesion resolved within 7-10 days of initiating endodontic treatment. 
DISCUSSION: The odontogenic cutaneous sinus tract on the facial and cervical skin often develops as a result of chronic apical periodontitis caused by pulpal degeneration or necrosis.6,7 The apical infection may spread through the marrow space, then perforate the cortical bone. In soft tissue, the infection may spread through the path of least resistance between facial spaces and finally perforate a mucosal or cutaneous surface. 6,8 However, Chan et al. reported an extraoral cutaneous sinus tract caused by vertical root fracture. ${ }^{6}$ Calıskan et al. also reported a case of cutaneous sinus tract originating from a fractured crown caused by trauma. ${ }^{9}$

Cutaneous sinus tracts most commonly present on the chin and the cheek area. ${ }^{8}$ They are rarely found in the nasal region. ${ }^{8}$ These tracts usually appear as suppurative lesions of the chin or neck. The inner surface of sinus tracts may be partially lined with either granulomatous tissue or epithelium. ${ }^{9}$

Cutaneous sinus tracts of dental origin have been well documented in the medical literature. However, these lesions continue to be a diagnostic dilemma. ${ }^{8}$ The differential diagnosis is of prime importance. The differential diagnosis should include traumatic lesions, fungal and bacteriologic infections, neoplasms, presence of foreign body, local skin infection, pyogenic granuloma, chronic tuberculosis lesion, osteomyelitis, actinomycosis and gumma of tertiary syphilis. Rare entities to be included in the differential diagnosis are defects of thyroglossal duct origin or branchial cleft, salivary gland and duct fistula and suppurative lymphadenitis.8,10-13 Illustrating the difficulty that can be encountered, a literature review revealed one case of cutaneous odontogenic sinus tract in which the correct diagnosis was made 32 years after the discharging lesion was first observed.14

Odontogenic cutaneous sinus tracts in the face and neck region are rare and present a diagnostic challenge to clinician as they may present a wide variety of diseases.6, 8 Misdiagnosis and delay in accurate treatment protocol may often be encountered.6,8 Therefore, when diagnosing and treating sinus tracts of unknown aetiology in the head and neck region, dermatologist or plastic surgeon should always consult dentists to rule out a dental cause even though there is no dental complaint.2, 6, 8 This is because the cutaneous sinus tract caused by chronic infection is often painless and may develop over a long period of time without alarming the patient.6,8 Patients rarely relate the symptoms to dental infection. Therefore, early and proper diagnosis is essential. An accurate diagnosis should include medical history of the patient, inspection and palpation of the lesion, pulp vitality test and intraoral radiographs. ${ }^{9}, 10$ In addition, the insertion of a probe or gutta-percha through the fistula to take radiographs is an effective method for determining the involved tooth. ${ }^{8}$ As suggested in literature, nonsurgical endodontic therapy is the treatment of choice of such lesions and should be attempted first. ${ }^{8}$

In all the cases described here, only nonsurgical endodontic therapy was carried out and the sinus tract was successfully treated with minimal scar formation. Calcium hydroxide was used as an intracanal medicament in all cases due to its beneficial effects. ${ }^{15}$ The advantages of calcium hydroxide treatment are stimulation of bone repair and bactericidal effects due to its high alkalinity. Usage of calcium hydroxide paste was advocated for rapid and successful treatment of sinus tract associated with necrotic teeth. ${ }^{15}$ After a long-term postendodontic observation period, surgical removal of the lesion was not necessary.

The principle of managing such lesions is to remove the source of dental infection. ${ }^{8}$ Conventional root canal treatment and occasionally periapical surgery and extraction are effective in providing disappearing sinus tract in a very short duration. $6,8,12,13,16$ 
CONCLUSION: When a draining lesion is encountered on the skin of the face, endodontic origin should always be considered in differential diagnosis. Non-surgical endodontic therapy, sometimes complimented by endodontic surgery or extraction is the choice of treatment in these cases. The present case report demonstrates non surgical conservative endodontic management which resulted in the elimination of a cutaneous sinus tract of dental origin in patients with a good host response.

\section{REFERENCES:}

1. Cantatore JL, Klein PA, Lieblich LM. Cutaneous dental sinus tract, a common misdiagnosis: a case report and review of the literature. Cutis; Cutaneous Medicine for the practitioner 2002; 70 (5): $264-265$.

2. Kaban L. Draining skin lesions of dental origin: the path of spread of chronic odontogenic infection. Plast Reconstr Surg.1980; 66: 711-717.

3. McWalters GM, Alexander JB, del Rio CE et al. Cutaneous sinus tracts of dental etiology. Oral Surg Oral Med Oral pathol. 1988; 66: 608-614.

4. Kotecha M, Browne MK. Mandibular sinuses of dental origin. Practitioner. 198; 225: 910-915.

5. Thoma KH. Oral Surgery, 4th edn. St Louis, USA: Mosby; 1963; 733.

6. Chan C.P., Chang S.H., Huang C.C., Wu SK. Cutaneous sinus tract caused by vertical root fracture. J Endod, 1997, 23(9): 593-595.

7. Hwang K., Kim C.W., Lee S.I. Cutaneous sinus tract from remaining tooth fragment of edentulous maxilla. J Craniofac Surg, 2000, 11: 254-257.

8. Heling E., Rotstein I. A persistent oronasal sinus tract of endodontic. J Endod, 1989, 15(3): 132134.

9. Calışkan M.K., Sen B.H., Ozinel M.A. Treatment of extraoral sinus tracts from traumatized teeth with apical periodontitis. Endod Dent Traumatol, 1995, 11: 115-120.

10. Wood N.K., Goaz P.W. Differential diagnosis of oral lesions. St. Louis. Mosby, 1975, 201.

11. Craig R.M., Andrews J.D., Wescott W.B. Draining fistulas associated with an endodontically treated tooth. J Am Dent Assoc, 1984, 108: 851-852.

12. Lewin-Epstein J., Taicher S., Azaz B. Cutaneous sinus tracts of dental origin. Arch Dermatol, 1978, 114: 1158-1161.

13. Spear K.L., Sheridan P.J., Perry H.O. Sinus tracts to the chin and jaw of dental origin. J Am Acad Dermatol, 1983, 8: 486-492.

14. Bernick SM, Jensen JR. Chronic draining extraoral fistula of 32 years duration. Oral Surg. 1969; 27: 790.

15. Gutmann J.L., Fava L.R.G. Periradicular healing and apical closure of a non-vital tooth in the presence of bacterial contamination. Int Endod J, 1992, 25: 307-311.

16. Alibhai M, Balasundaram I, Bridle C. Cutaneous sinuses of dental origin. JSCR. 2012, 8:6. 


\section{CASE REPORT}

\section{CASE 1: FIGURE 1}

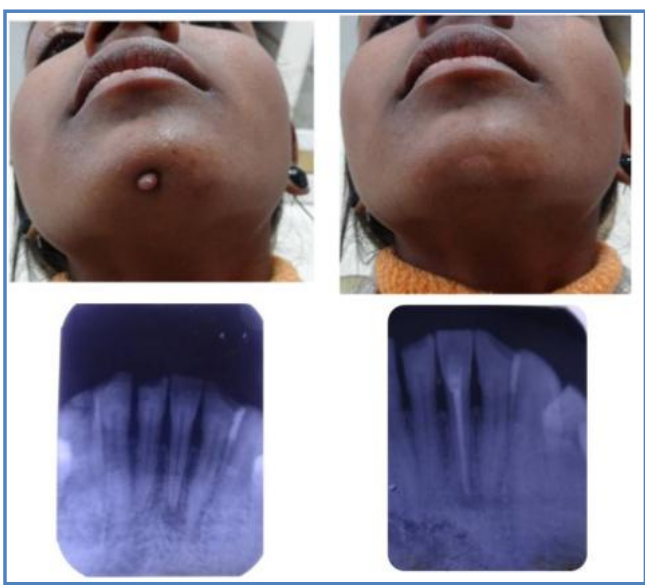

CASE 2: FIGURE 2

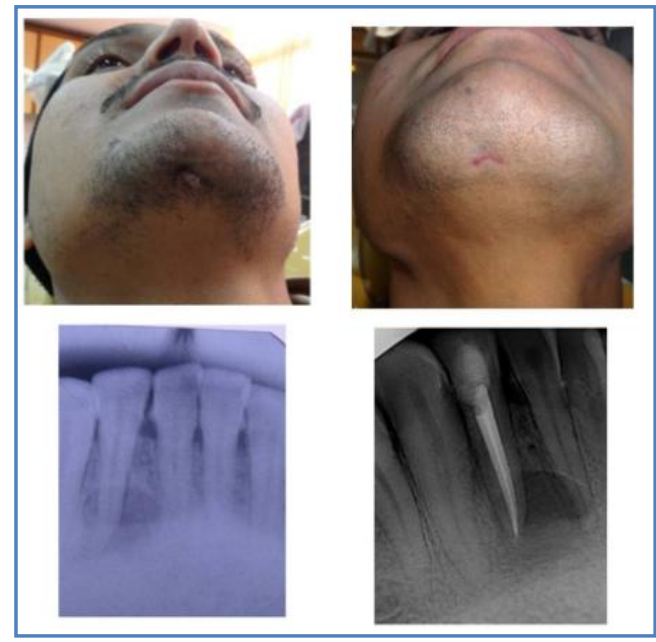

\section{CASE 3: FIGURE 3}

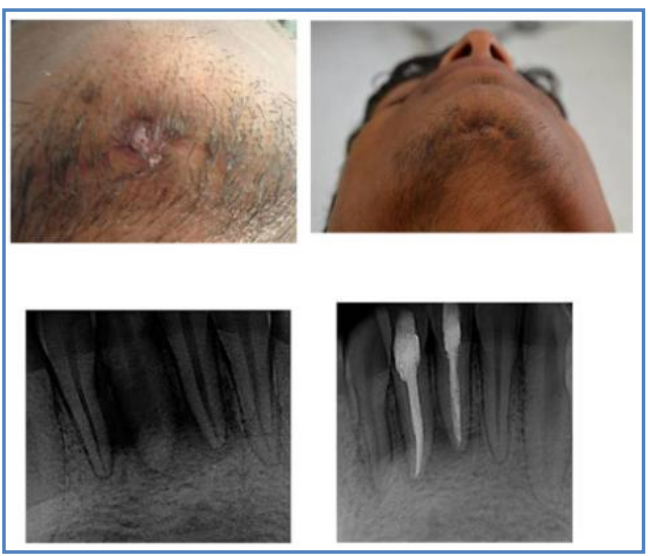

Figure 1 : Preoperative photograph showing extraoral draining sinus opening, preoperative radiograph showing 42 with radiolucent lesion, one month post operative photograph showing healed lesion, radiograph one month after obturation.
Figure 2 : Preoperative photograph showing extraoral draining sinus opening, preoperative radiograph showing 31,41 with radiolucent lesion, two months post operative photograph showing healed lesion, radiograph two months after obturation.
Figure 3 : Preoperative photograph showing extraoral draining sinus opening, preoperative radiograph showing 31 with radiolucent lesion, 10 days post operative photograph showing healed lesion, radiograph days after obturation. 


\section{AUTHORS:}

1. Rohit Kansal

2. Aishvarya Kaushik

3. Sangeeta Talwar

4. Sarika Chaudhary

5. Ruchika Nawal

\section{PARTICULARS OF CONTRIBUTORS:}

1. Post Graduate Student, Department of Conservative Dentistry and Endodontics, Maulana Azad Institute of Dental Sciences, New Delhi.

2. Post Graduate Student, Department of Conservative Dentistry and Endodontics, Maulana Azad Institute of Dental Sciences, New Delhi.

3. Professor \& HOD, Department of Conservative Dentistry and Endodontics, Maulana Azad Institute of Dental Sciences, New Delhi.
4. Associate Professor, Department of Conservative Dentistry and Endodontics, Maulana Azad Institute of Dental Sciences, New Delhi.

5. Associate Professor, Department of Conservative Dentistry and Endodontics, Maulana Azad Institute of Dental Sciences, New Delhi.

\section{NAME ADDRESS EMAIL ID OF THE}

\section{CORRESPONDING AUTHOR:}

Dr. Rohit Kansal,

AK - 87, Shalimar Bagh,

New Delhi.

Email - rohitkansal20@gmail.com

Date of Submission: 02/11/2013.

Date of Peer Review: 05/11/2013.

Date of Acceptance: 12/11/2013.

Date of Publishing: 15/11/2013 